\title{
Advances in Electrochemical Models for Predicting the Cycling Performance of Traction Batteries: Experimental Study on Ni-MH and Simulation
}

\author{
J. Bernard' ${ }^{1}$, A. Sciarretta ${ }^{2}$, Y. Touzani ${ }^{2}$ and V. Sauvant-Moynot ${ }^{1}$ \\ 1 Institut français du pétrole, IFP-Lyon, Rond-point de l'échangeur de Solaize, BP 3, 69360 Solaize - France \\ 2 Institut français du pétrole, IFP, 1-4 avenue de Bois-Préau, 92852 Rueil-Malmaison Cedex - France \\ e-mail : julien.bernard@ifp.fr - antonio.sciarretta@ifp.fr - youssef.touzani@ifp.fr - valerie.sauvant@ifp.fr
}

\begin{abstract}
Résumé - Développement de modèles électrochimiques de batteries de traction pour la prédiction de performances : étude expérimentale de batteries NiMH et simulations - Des modèles électrochimiques fins permettant de simuler le comportement de batteries ont été développés avec succès et reportés dans la littérature. Ils constituent une alternative aux méthodes classiques pour estimer l'état de charge (SoC pour State of Charge) des batteries, cette variable étant ici un paramètre interne du modèle physique. Cependant, pour les applications embarquées, il est nécessaire de développer des modèles simplifiés sur la base de ces modèles physiques afin de diminuer le temps de calcul nécessaire à la résolution des équations. Ici, nous présenterons à titre d'exemple un modèle électrochimique 0D avancé d'un accumulateur NiMH et sa validation. Ce modèle à paramètres concentrés sera utilisé pour réaliser un filtre de Kalman qui permettra la prédiction de l'état de charge d'un pack complet. Une étude expérimentale d'accumulateurs NiMH permettra de mieux comprendre les mécanismes physico-chimiques ayant lieu à chaque électrode et ainsi d'alimenter le modèle physique en informations. La dernière partie de cet article sera consacrée à la validation du modèle par comparaison à des données expérimentales obtenues sur cellule individuelle mais également sur un pack batterie NiMH commercial complet.
\end{abstract}

\begin{abstract}
Advances in Electrochemical Models for Predicting the Cycling Performance of Traction Batteries: Experimental Study on Ni-MH and Simulation - Rigorous electrochemical models to simulate the cycling performance of batteries have been successfully developed and reported in the literature. They constitute a very promising approach for State-of-Charge (SoC) estimation based on the physics of the cell with regards to other methods since SoC is an internal parameter of these physical models. However, the computational time needed to solve electrochemical battery models for online applications requires to develop a simplified physics-based battery model. In this work, our goal is to present and validate an advanced OD-electrochemical model of a Ni-MH cell, as an example. This lumped-parameter model will be used to design an extended Kalman filter to predict the SoC of a Ni-MH pack. It is presented, followed by an extensive experimental study conducted on Ni-MH cells to better understand the mechanisms of physico-chemical phenomena occurring at both electrodes and support the model development. The last part of the paper focuses on the evaluation of the model with regards to experimental results obtained on $\mathrm{Ni}-\mathrm{MH}$ sealed cells but also on the related commercial HEV battery pack.
\end{abstract}




\section{INTRODUCTION}

The technical and commercial success of Hybrid-Electric Vehicles (HEV) is strongly related to the effectiveness of the vehicle supervisory controller that manages the power flows between the multiple energy sources. Regardless of the control strategy adopted, one essential information to the supervisor is the state of the energy storage system. This quantity related to the concentration of reactive species inside the battery cells is not directly measurable on board. Given the very specific environment of traction batteries $[1,2]$ used in HEV possibly highly dynamic and power demanding (current usually reaches intensive C-rates in charge or discharge up to $\pm 20 \mathrm{C}$ ), in addition covering a large temperature range, $\mathrm{SoC}$ estimation is a rather difficult task.

Numerous approaches have been studied to monitor the State-of-Charge (SoC) of a cell $[3,4]$. The coulomb-counting procedure is not reliable for a precise SoC determination in traction batteries since not all current supplied goes to charging the cell and the charge lost to any undesired charge reaction is not included. In order to take into account the typical battery usage in an automotive context, more precise techniques of estimation are required. In this framework, a clear trend in the last years is toward the use of mathematical models of the battery systems to inspire ad-hoc estimation strategies.

Among the most used method of SoC determination, the parameterization of the battery electro-motive force (emf) as a function of $\mathrm{SoC}$ could in principle provide information on the $\mathrm{SoC}$ from an estimation or a measurement of the opencircuit voltage. In particular, the emf of Li-ion battery in combination with the coulomb-counting approach has been demonstrated to be a good indication of SoC $[5,6]$, since the emf-SoC dependency does not change with cycling, if the $\mathrm{SoC}$ is always referred to the actual battery capacity. In contrast, there is no direct relationship between emf and $\mathrm{SoC}$ for $\mathrm{Ni}-\mathrm{MH}$, since a typical hysteresis phenomenon is prominent in this case. Another main drawback of this estimation technique is that the measurement of the emf needs long rest time, during which the battery is not yielding current which is hardly achievable in HEV operation.

Methods employing electrical circuit analogy are also widely studied. Electrical models are electrical equivalent models made of a combination of voltage sources, resistors and capacitors. Equivalent-circuit models can be further differentiated between black-box models in the form of lumped-parameter dynamic electric equivalent circuits, and grey-box models, still in the form of equivalent electric circuits, but where the circuit elements try to reproduce the inner electrochemical behavior of the cells. Many electrical models of batteries have been presented in the literature, for lead-acid [7], Ni-MH [8, 9] and Lithium-ion batteries [10], since they are intuitive, easy to handle and useful as battery simulator to design hybrid architecture [11]. Those models rely on the collection of appropriate look-up tables from cell data. This requires generating enough test points to create a series of curves which can be broken down into intervals to estimate the curvature with a stepwise approximation. This method cannot offer the best accuracy to estimate the SoC while dealing with spread in both battery and user behaviour, large temperature and current range, and ageing of the cells under all realistic user conditions. In addition, equivalent circuit models are tracking a parameter to estimate the $\mathrm{SoC}$ which has often little physical significance. In conclusion, the results of electric equivalent circuit models are nowadays of limited value in predicting the SoC of a HEV battery online.

Electrochemical models, as an alternative approach to equivalent circuit based models, characterise the fundamental mechanisms of power generation and relate battery design parameters with macroscopic (e.g. battery voltage and current) and microscopic (e.g. concentration distribution) information. They constitute a very promising approach with regards to other methods since $\mathrm{SoC}$ is an internal parameter of these physical models. Sophisticated and simpler models were presented as valuable physical models to predict the $\mathrm{SoC}$ and other performance of both $\mathrm{Ni}-\mathrm{MH}$ cells [12-16] and lithium ion cells [17-20]. However, the computational time needed to solve rigorous electrochemical battery models for online applications requires to develop a simplified physics-based battery model with specific efforts to account for the diffusion phenomena. Those later are not taken into account yet in 0D-model, which alters their predictability in power applications [21].

The objective of this paper is to present and validate an advanced 0D-electrochemical model of a Ni-MH cell. Attempts are made to extend the model to describe a battery pack as well. This lumped-parameter model will be used in future work to design an extended Kalman filter to predict the SoC of a Ni-MH cell. The second part of the paper is dedicated to the development of an advanced lumped-parameter electrochemical model in the AMESim simulation environment, with specific efforts to account for the diffusion phenomena. An extensive experimental study conducted on sealed Ni-MH cells to better understand the mechanisms of physico-chemical phenomena occurring at both electrodes, and support the model development is presented in the first part of the paper. Electrochemical Impedance Spectroscopy (EIS) with a 3-electrode set-up has been used for this purpose over a large temperature range $\left(-20^{\circ} \mathrm{C}\right.$ to $35^{\circ} \mathrm{C}$ ), pointing out the large contribution of diffusion phenomena. The last part focuses on the comparison between experimental and simulation results, looking at charge and discharge curves obtained on Ni-MH cells but also on EIS spectra thanks to an original co-simulation model (AMESim/Simulink). The discussion is completed with regards to experimental data collected on a commercial HEV pack as well. 


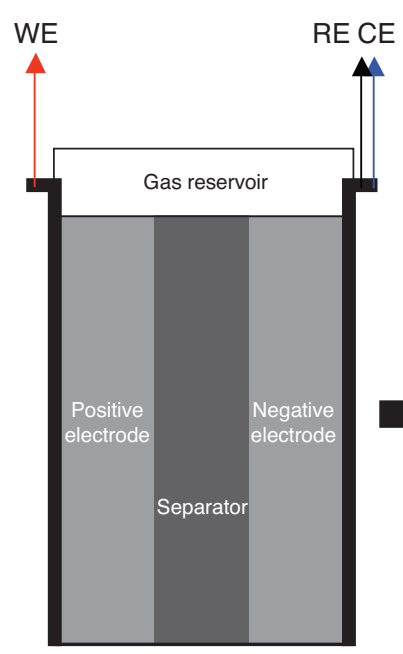

a)

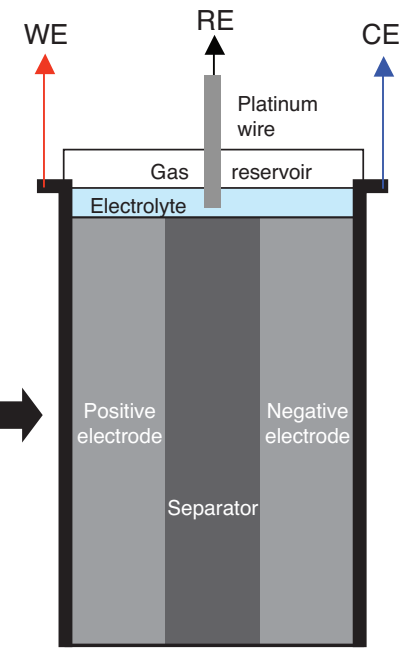

b)
Figure 1

Schematic view of a commercial NiMH cell a) before modification and b) after insertion of the quasi-reference electrode.

\section{EXPERIMENTAL}

The experimental work was performed on a commercial HEV Nickel/metal hydride battery pack (168 cells, $202 \mathrm{~V}$ nominal, 6.5 Ah). Sealed Ni-MH cells (1.2 V nominal, 6.5 Ah) were extracted from the battery pack to conduct charge/ discharge experiments. A sealed nickel/metal hydride battery cell is composed of four regions (Fig. Ia): a negative electrode (Metal Hydride MH), a positive electrode (porous nickel oxyhydroxide $\mathrm{NiOOH}$ ), a separator (porous nylon material, typically) and a gas reservoir. Both electrodes are electronic conducting porous materials with constant porosity flooded with the electrolyte (concentrated $\mathrm{KOH}$ aqueous solution) which fully impregnates the separator as well. Each cell was characterised in terms of capacity and physical parameters such as electrode and separator nature, thicknesses and electrolyte concentration to provide related parameters for the simulation and try to duplicate exactly the runs with the model. However, other microscopic geometrical parameters were selected according to the literature [13, 22]. Under this hypothesis, nickel oxide electrode consists of composite cylindrical needles with a substrate inside. The MH electrode $\left(\mathrm{LaNi}_{5}\right)$ consists of spherical particles (powders with uniform size) of the metal-hydride material only, without any deposited metal layer on their surface.

Charge/discharge cycling on a cell was performed with a VMP3 multi-channel potentiostat from Biologic Science Instruments. Tests were conducted in a climatic chamber allowing measurements from $-40^{\circ} \mathrm{C}$ to $180^{\circ} \mathrm{C}$. Each channel is equipped with the Electrochemical Impedance Spectroscopy (EIS) option allowing independent EIS measurements on each channel. EIS consists of the application of a current/voltage excitation waveform to the system and the monitoring of the system's voltage/current response. In this work, all EIS measurements were done in a galvanostatic mode. The current amplitude was adapted to obtain a potential amplitude response under $10 \mathrm{mV} \mathrm{cc}$. This amplitude and also the stationarity of the response signal were controlled using an oscilloscope. Impedance diagrams were plotted between $10 \mathrm{kHz}$ and $50 \mathrm{mHz}$.

The application of EIS with a 3-electrode set-up to Ni-MH cell in function of current, temperature and during representative charge cycles will help to determine electrochemical phenomena occurring at both electrodes [23]. To build a 3 -electrode set-up, a hole was made in the upper part of the cell and half of the gas reservoir was filled with an electrolytic solution $(\mathrm{KOH} 6 \mathrm{~mol} / \mathrm{L})$. A platinum wire was then inserted in the hole and immersed in the electrolyte, a part of the wire standing higher than the top of the cell to allow an external connection (Fig. 1b). The platinum wire immersed in a $\mathrm{KOH}$ solution with constant concentration constitutes a quasi-reference electrode. The stability of its potential versus a commercial $\mathrm{Hg} / \mathrm{HgO}$ was verified in isothermal conditions. Finally, a resin was used to fix the platinum wire and ensure that the cell was sealed.

Different connection types were used:

- the complete cell was studied with a 2-electrode configuration: the Working Electrode (WE) gives the potential of positive electrode, the Reference Electrode (RE) and the Counter Electrode (CE) are connected to the negative;

- the positive electrode was investigated with a 3-electrode configuration: $\mathrm{WE}$ to the positive, $\mathrm{RE}$ to the platinum wire and $\mathrm{CE}$ to the negative;

- the negative electrode was investigated with a 3-electrode configuration as well: WE to the positive, RE to the platinum wire and $\mathrm{CE}$ to the positive.

Charge/discharge cycles following the Hybrid Pulse Power Characterisation test [24] (HPPC) were also applied on the full battery pack which contains 168 sealed Ni-MH cells connected in series. A Digatron $500 \mathrm{~A} / 500 \mathrm{~V}$ test bench was used for this purpose at $20^{\circ} \mathrm{C}$. The HPPC test is proposed in the FreedomCar US Department of Energy program to demonstrate the discharge pulse and regenerative pulse power capabilities of a HEV battery at various Depth of Discharge (DoD) values for both the Minimum and Maximum Power-Assist goals.

\section{FORMULATION OF THE OD-MODEL}

A Ni-MH cell is a dual-intercalation electrochemical system in which proton insertion in the positive solid nickel electrode and hydrogen de-insertion in the negative metal/hydride 
electrode occur during discharge and vice versa during charge. Electrochemical reactions taking place at the electrode/electrolyte interface (assuming discharge) are:

$$
\begin{gathered}
\mathrm{NiOOH}(s)+\mathrm{H}_{2} \mathrm{O}(l)+\mathrm{e}^{-} \leftrightarrow \mathrm{Ni}(\mathrm{OH})_{2}(s)+\mathrm{OH}^{-}(a q) \\
1 / 2 \mathrm{O}_{2}+\mathrm{H}_{2} \mathrm{O}+2 \mathrm{e}^{-} \leftrightarrow 2 \mathrm{OH}^{-}
\end{gathered}
$$

at the positive electrode and:

$$
\begin{array}{r}
\mathrm{M}(s)+\mathrm{H}_{2} \mathrm{O}(l)+\mathrm{e}^{-} \leftrightarrow \mathrm{MH}(s)+\mathrm{OH}^{-}(a q) \\
1 / 2 \mathrm{O}_{2}+\mathrm{H}_{2} \mathrm{O}+2 \mathrm{e}^{-} \leftrightarrow 2 \mathrm{OH}^{-}
\end{array}
$$

at the negative electrode.

Side Equations (2) and (4) constitute the oxygen recirculation inside the cell. The oxygen is transported from one electrode to the other via the liquid electrolyte and, after exceeding its solubility limit in the electrolyte, via a gas phase. Thus a $\mathrm{Ni}-\mathrm{MH}$ cell is a three-phase system, with one gas phase besides the solid matrices and the liquid electrolyte. The accumulation of oxygen in the gas phase increases the cell pressure in the gas reservoir above the cell (constant volume).

The modelling of such a system can be very complex if the spatial distribution of all the species and phases are represented, since the relevant mass and energy conservation laws have to be applied locally for a three-dimensional domain. Several techniques have been proposed in order to reduce the complexity of this approach. The pseudo-twodimensional method $[16,25]$ considers spatial variations of concentrations, potentials, etc., along a main spatial $x$-direction and two fictitious $y$-directions. The main $x$-axis encompasses the two porous electrodes and the separator and it is bounded by the battery terminal plates. The two $y$-axes represent diffusion within fictitious solid particles in each electrode. These fictitious particles have a spherical geometry in the $\mathrm{MH}$ electrode and a cylindrical geometry in the nickel electrode. A main simplification of this approach (diffusion length approach) consists in neglecting the diffusion along the $y$-directions, except for a localized concentration discontinuity between the bulk of the fictitious particle and its interface with the electrolyte. Computationally, the model thus reduces to a one-dimensional (1D) domain [13].

Even if simplified to a single dimensional domain, distributedparameter electrochemical battery models are of limited value in predicting the $\mathrm{SoC}$ of a battery on-board due to the excessive computational time required. Nevertheless, besides being useful for physical understanding and diagnostics, they can be used to assess the prediction capability of a simpler but computationally efficient approach, the so called lumpedparameter electrochemical model.

The lumped-parameter description is based on the assumption of considering the concentrations of the five species as homogeneous within the battery, i.e., the planar electrode approximation $[14,15]$. The reduction of the number of state variables is usually achieved with a simplification that consists of evaluating the concentration of $\mathrm{NiOOH}$ as a function of nickel hydroxide concentration. Moreover, the concentration of the $\mathrm{OH}^{-}$ions in the electrolyte is taken as a constant. Consequently, the model state equations are given by the mass balance equations of the three remaining species. The mass flows involving the three species are related to the current densities of the main and side reactions shown above. The four current densities are calculated using the ButlerVolmer equations as a function of the species concentrations, the potential differences at the solid-liquid interface on the electrodes, and the equilibrium potentials (open-circuit potentials) of the reactions. Current balance equations provide the missing relationships between the reaction current densities, the potential differences, the cell voltage, and the cell current. Moreover, the equilibrium potential of the nickel reaction can be calculated in such a way to account for the typical hysteresis effect of Ni-MH batteries.

A comparison between 1D and 0D electrochemical models taken from the literature for a Ni-MH battery cell has been recently presented, including predictions of the terminal voltage along a given current profile [21].

Although the main cell dynamics are qualitatively reproduced by the $0 \mathrm{D}$ model, nevertheless the comparison has shown that, contrarily to what often stated in the literature, the $0 \mathrm{D}$ approach introduces substantial quantitative differences with respect to its $1 \mathrm{D}$ counterpart. These differences are mainly due to the fact that literature 0D models renounce representing the diffusion processes. Indeed, literature 0D models only include three state variables for a single cell, while a typical 1D model requires more than thirty dynamic variables.

\subsection{Assumptions}

An improved electrochemical lumped-parameter model for a sealed Ni-MH cell is proposed in order to better approach the outcome of 1D models, while keeping the same level of complexity of standard 0D models. The main assumptions are listed below:

- the concentration of active species is homogeneous in each of the cell regions, i.e., spatial gradients are neglected. Thus the concentrations of active species, namely hydrogen in metal hydride material, hydrogen in the $\mathrm{Ni}(\mathrm{OH})_{2}$ material), oxygen dissolved in the liquid phase, gaseous oxygen and electrolyte are only functions of time;

- a double-layer capacitance is introduced in the charge balance equation to account for the electrolyte ionic species accumulation at both electrode interfaces due to the diffusion gradient;

- the diffusion into a single solid particle is accounted for by an equation based on the superposition integral [12]. Then the bulk concentration is distinguished from the interfacial concentration for each species, both being functions of time; 
- liquid/gas interfacial equilibrium for the oxygen species links the dissolved oxygen concentration to the gaseous oxygen pressure via the Henry law, while the ideal gas law applies in the reservoir;

- potential hysteresis is considered;

- thermal effects are adequately taken into account by introducing an energy balance equation for the whole cell. Heat production due to irreversibility in the reactions, heat losses through the cell external surface, as well as energy accumulation in the cell are the terms of such equation. Moreover, cell temperature affects the reaction kinetics.

\subsection{Equations}

The kinetics of reactions (1-4) are derived from the general Butler-Volmer equations with respect to a specified reference state:

$$
\begin{gathered}
J_{1}(t)=J_{1,0}\left\{\left(\frac{c_{n}(t)}{c_{n, r e f}}\right)\left(\frac{c_{e}}{c_{e, r e f}}\right) e^{0.5 K \eta_{1}(t)}-\right. \\
\left.\left(\frac{c_{n, \text { max }}-c_{n}(t)}{c_{n, \text { max }}-c_{n, \text { ref }}}\right) e^{-0.5 K \eta_{1}(t)}\right\} \exp \left(\frac{E_{a, 1}}{R}\left(\frac{1}{T}-\frac{1}{T_{0}}\right)\right) \\
J_{2}(t)=J_{2,0}\left\{\left(\frac{c_{e}}{c_{e, r e f}}\right)^{2} e^{1.5 K \eta_{2}(t)}-\right. \\
\left.\left(\frac{\bar{c}_{0}(t)}{c_{0, r e f}}\right)^{\frac{1}{2}} e^{-0.5 K \eta_{2}(t)}\right\} \exp \left(\frac{E_{a, 2}}{R}\left(\frac{1}{T}-\frac{1}{T_{0}}\right)\right) \\
\left.J_{3}(t)=J_{3,0}\left(\frac{c_{m}}{c_{m, r e f}}\right)^{\mu}\left(\frac{c_{e}}{c_{e, r e f}}\right) e^{0.5 K \eta_{3}(t)}-e^{-0.5 K \eta_{3}(t)}\right\} \\
\times \exp \left(\frac{E_{a, 3}}{R}\left(\frac{1}{T}-\frac{1}{T_{0}}\right)\right) \\
J_{4}(t)=J_{4,0}\left\{\left(\frac{c_{e}}{c_{e, r e f}}\right)^{2} e^{1.5 K \eta_{4}(t)}-\right. \\
\left.\left(\frac{\bar{c}_{0}(t)}{c_{0, r e f}}\right)^{\frac{1}{2}} e^{-0.5 K \eta_{4}(t)}\right\} \exp \left(\frac{E_{a, 4}}{R}\left(\frac{1}{T}-\frac{1}{T_{0}}\right)\right)
\end{gathered}
$$

where $c_{n}$ is the proton interfacial concentration in nickel hydroxide, $c_{e}$ is the constant concentration of $\mathrm{KOH}$ electrolyte representing the concentration of $\mathrm{OH}^{-}$ions, $c_{0}$ is the concentration of dissolved oxygen, $c_{m}$ is the interfacial concentration of hydrogen in metal hydride material and $\mu$ its stoichiometric coefficient. The latter is variously calculated in the literature, and corresponds to $1 / 6$ for $\mathrm{LaNi}_{5} \mathrm{H}_{6}$ [13]. In the above equations, $\eta$ 's with various superscripts stand for the surface overpotentials:

$$
\begin{aligned}
& \eta_{1}(t)=\Delta \Phi_{p o s}(t)-U_{\text {eq,ref } 1}(t) \\
& \eta_{2}(t)=\Delta \Phi_{p o s}(t)-U_{\text {eq,ref } 2}(t) \\
& \eta_{3}(t)=\Delta \Phi_{\text {neg }}(t)-U_{\text {eq,ref } 3}(t) \\
& \eta_{4}(t)=\Delta \Phi_{\text {neg }}(t)-U_{\text {eq,ref } 4}(t)
\end{aligned}
$$

where $\Delta \Phi_{p o s}$ and $\Delta \Phi_{n e g}$ are the potential differences at the solid-liquid interface on the positive and negative electrodes, respectively. The latter terms are conveniently parameterised as a function of the species concentrations and the temperature. The typical hysteresis behavior of Ni-MH batteries can be conveniently simulated by differentiating $U_{\text {eq,ref,1 }}$ between charging and discharging and relaxing the switching with an exponential term:

$$
\begin{aligned}
& \frac{d U_{e q, r e f, 1}}{d t}=\left(U_{\text {eq, }, \text { ee }, 1, \infty}-U_{\text {eq, }, \text { ef }, 1}\right) \cdot k(I) \cdot|I| \\
& k(I)= \begin{cases}k_{c}, & I>0 \\
k_{d}, & I<0\end{cases} \\
& U_{\text {eq, }, \text { ef }, 1, \infty}= \begin{cases}U_{\text {eq, ref }, 1, c}, & I>0 \\
U_{\text {eq }, \text { ref }, 1, d}, & I<0\end{cases}
\end{aligned}
$$

For example, the values used in Reference [15] are $U_{\text {eq, }, e f, 1, d}=0.427$ for discharging and $U_{e q, r e f, 1, c}=0.527$ for charging.

Conservation equations in the lumped-parameter approach are derived from traditional transport equations modelled using concentration solution theory [26]. The basis of concentrated solution theory is that the gradient in the electrochemical potential is the driving force for mass transfer. The mass concentration of species $i$ in a general form is written as:

$$
\varepsilon_{(k)} \frac{\partial c_{i}}{\partial t}=-\nabla N_{i}+R_{i}
$$

where $c_{i}$ is the concentration of the $i$-th species, $N_{i}$ its flux density $\left(\mathrm{mol} /\left(\mathrm{cm}^{2} . \mathrm{s}\right)\right)$, and $R_{i}$ its generation rate $\left(\mathrm{mol} /\left(\mathrm{cm}^{3} . \mathrm{s}\right)\right)$.

Applying the general volume-averages conservation equation of species and making use of the interfacial balance condition to active species in the finite-volume approach yield:

$$
\varepsilon_{e} \frac{d c_{e}}{d t}=0
$$

for the electrolyte concentration, which is constant in the OD-approximation:

$$
\begin{gathered}
\frac{d c_{m}}{d t}=-\frac{2}{r} \frac{J_{3}}{F} \\
\frac{d c_{n}}{d t}=-\frac{1}{y_{(1)}} \frac{J_{1}}{F}
\end{gathered}
$$

where $r$ is the radius of the $\mathrm{MH}$ particles (mass conservation written in a cylindrical geometry) and $y_{(1)}$ is the thickness 
of active materials in the nickel electrode surrounding the substrate along the central axis of the cylinders.

To take into account in a lumped-parameter way the diffusion in the solid phase, the concentrations calculated with Equations (13-14) can be distinguished from the concentrations used in the Butler-Volmer equations. Inspired by the diffusion length approach where the diffusion rate is constant along a diffusion length $l_{s e}$ across the characteristic geometry [13], the rules to differentiate the average concentration from the interfacial concentration are:

$$
\begin{gathered}
\bar{c}_{n}=c_{n}-\frac{l_{s e} J_{1}}{F D_{h}} \\
\bar{c}_{m}=c_{m}-\frac{l_{s e} J_{3}}{F D_{h}}
\end{gathered}
$$

where $D_{h}$ is the diffusion coefficient of hydrogen, $c_{i}$ is the average concentration of species $i$ and $c_{i}$ with bar is the interfacial concentration, which is also used in the Butler-Volmer Equations (5) to (8).

The concentration of the oxygen in the gaseous phase is averaged in (17) as in literature Reference [13], while the concentration of oxygen in the liquid phase is assumed to vary in a quasistatic way, thus:

$$
\begin{gathered}
\frac{d}{d t} p_{0}(t)=-\frac{R v_{b}}{V_{g}} \frac{A_{(1)} l_{(1)} a_{(1)} J_{2}(t)+A_{(3)} l_{(3)} a_{(3)} J_{4}(t)}{F} \\
c_{0(k)}(t)=\bar{c}_{0}(t)+\frac{1}{4 F K_{\text {tran }}} \begin{cases}a_{(1)} J_{2}(t) & k=1 \\
0 & k=2 \\
a_{(3)} J_{4}(t) & k=3\end{cases}
\end{gathered}
$$

with the latter being used in the Butler-Volmer Equations (6), (8).

The way proposed to include the effect of the double-layer capacitance on the electrode kinetics is as follows. Equation (5) is substituted by (19):

$$
J_{1}=f\left(\eta_{1}, c_{i}\right)+C_{(1)} \frac{d \eta_{1}}{d t}
$$

where $f(\cdot)$ is the right-hand side of Equation (5). Similarly, (7) is substituted by (20):

$$
J_{3}=g\left(\eta_{3}, c_{i}\right)+C_{(3)} \frac{d \eta_{3}}{d t}
$$

where $g(\cdot)$ is the right-hand side of Equation (7). The two capacitances per surface unit $C_{(1)}$ and $C_{(3)}$ might be different in principle. The model is completed by a voltage balance across the cell including the Ohmic resistance (mainly of the electrolyte), $R_{\text {int }}$ :

$$
V(t)=\Delta \Phi_{p o s}(t)-\Delta \Phi_{n e g}(t)+R_{\mathrm{int}} I(t)
$$

Thus, the system is described by the five non-linear differential Equations (13), (14), (17), (19), and (20) in the five unknowns $c_{m}, c_{n}, p_{0}, \Delta \Omega_{\text {pos }}$ and $\Delta \Omega_{\text {neg }}$. Algebraic equations like (18) and (5-8) introducing new intermediate variables complete the model.

Temperature can be calculated from an energy balance of the whole cell. The heat flux generated by the cell is given by:

$$
\begin{aligned}
\varphi_{g e n}(t)=\sum_{z} & J_{z}(t)\left(U_{e q, r e f, z}(t)-T(t) \frac{d U_{e q, r e f, z}(t)}{d T}\right) A_{(z)} \\
& -V(t) I(t)
\end{aligned}
$$

where the term $U_{\text {eq,ref, } z}-V$ can be associated to the irreversible losses for each reaction $z$, while the reversible generation term $T \times d U_{\text {eq, ref }, 2} d T$ is associated to entropy variations. On the other hand, the heat flux to the ambient is given by Newton's law of cooling:

$$
\varphi_{\text {tra }}(t)=h A_{\text {cell }}\left(T(t)-T_{a}\right)
$$

where $h$ is a transfer coefficient related to convection and radiation, $T_{a}$ is the ambient temperature, and $A_{c e l l}$ is the cell surface. The cell energy conservation is thus written as:

$$
M_{\text {cell }} C_{p} \frac{d T(t)}{d t}=\varphi_{\text {gen }}(t)-\varphi_{\text {tra }}(t)
$$

where $C_{p}$ is the specific thermal capacity of the cell and $M_{\text {cell }}$ its mass. Note that certain cell parameters, such as internal resistance, diffusion coefficients, etc., are usually dependent on the temperature.

The link between species concentration and $\mathrm{SoC}$ is given as a double integral equation whose finite-volume counterpart is:

$$
q=-\frac{c_{n, \max }-c_{n}}{c_{n, \max }}
$$

\section{RESULTS}

\subsection{EIS}

Figure 2 shows the impedance responses of the entire cell (2-electrode measurement) and of both electrodes (3-electrode measurement) of a commercial Ni-MH cell modified with a reference electrode as describe previously $(\mathrm{SoC}=50 \%)$. To check the validity of the measurement the sum of the halfcell impedance results and the two-electrode measurements were compared.

For the entire cell a single semi-circle in the medium frequency range and a straight line in the low frequency range can be distinguished. According to Figure 2, the semicircle is due to the contribution of the negative electrode. The starting point of this semi-circle on the abscissa axis fits the internal resistance of the battery (or $R_{H F}$ for high frequency resistance). The semi-circle shape is typical of a charge transfer resistance in parallel with the double layer capacitance. The beginning of the line in low frequencies is principally controlled by the positive electrode. This line 


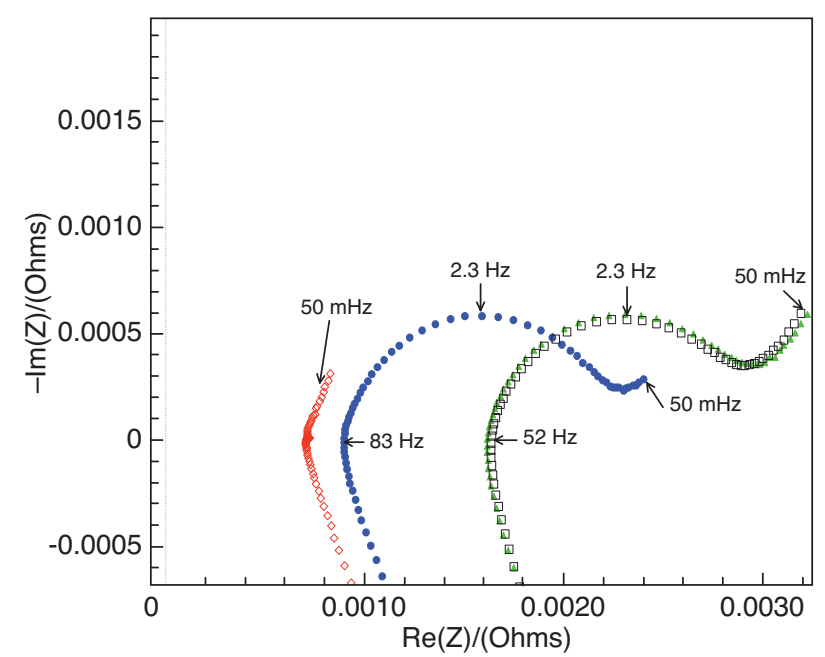

Figure 2

EIS diagrams of a NiMH commercial cell modified with a reference electrode at $\mathrm{SoC}=50 \%, 20^{\circ} \mathrm{C}$. Positive (red diamonds), negative (blue circles), entire cell (black rectangles), positive + negative (green triangles).

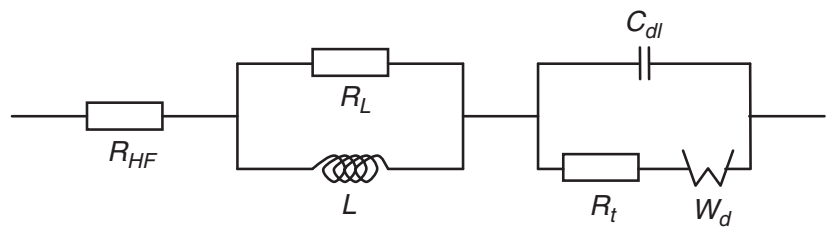

Warburg : $Z(f)=R_{d} \frac{\operatorname{th}\left(\sqrt{t_{d} j 2 \pi f}\right)}{\left.\sqrt{t_{d} j 2 \pi f}\right)}$

Figure 3

Equivalent circuit for the negative electrode of the NiMH cell and expression of the Warburg impedance for semi-infinite diffusion.

reflects the diffusion processes that can be modeled by a Warburg impedance with a $45^{\circ}$ slope.

To extract parameters of use in the model, EIS diagrams can be fitted with electrical equivalent circuits. For the

TABLE 1

Parameters of the equivalent circuit of the negative electrode

\begin{tabular}{c|c}
\hline$R_{H F}$ & $0.977 \times 10^{-3} \Omega$ \\
\hline$C_{d l}$ & $45.73 \mathrm{~F}$ \\
\hline$R_{t}$ & $0.946 \times 10^{-3} \Omega$ \\
\hline$R_{d}$ & $1.234 \times 10^{-3} \Omega$ \\
\hline$t_{d}$ & $81.14 \mathrm{~s}$ \\
\hline$L$ & $0.127 \times 10^{-6} \mathrm{H}$ \\
\hline$R_{L}$ & $1.391 \times 10^{-3} \Omega$ \\
\hline
\end{tabular}

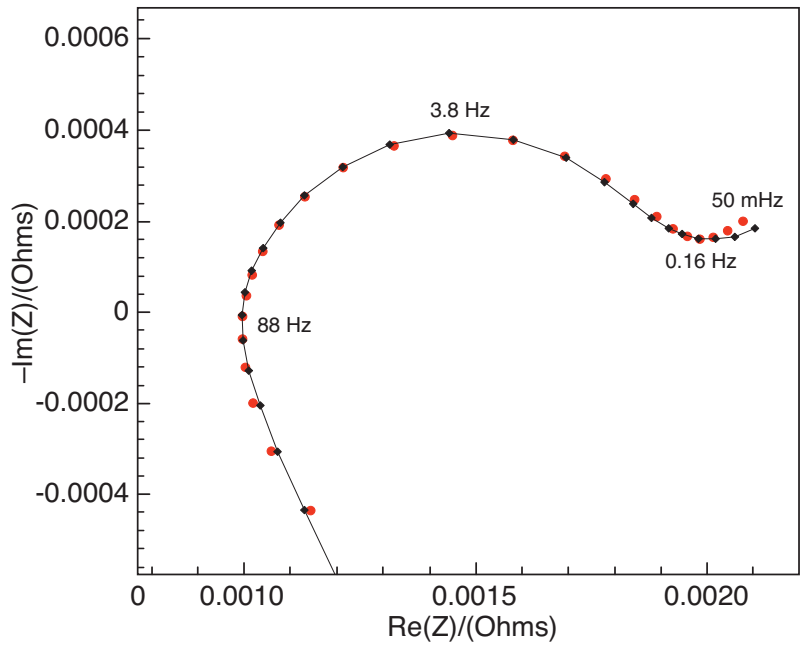

Figure 4

EIS diagram of a negative electrode (line + diamonds) at $\mathrm{SoC}=50 \%, 20^{\circ} \mathrm{C}$ and EIS diagram of the equivalent circuit (red circles).

negative electrode, a classical Randles circuit can be used (Fig. 3). The Randles circuit is composed of a double layer capacitance $\left(C_{d l}\right)$ in parallel with a charge transfer resistance $\left(R_{t}\right)$ and a Warburg impedance $\left(W_{d}\right)$. This circuit is completed with a high frequency resistance representing the internal resistance of the cell and a parallel RL circuit attributed to the inductive effects due to the electrode geometry and the cell connections. The EIS diagram fitting using parameters given in Table 1 is depicted in Figure 4. The double layer capacitance of the negative electrode is estimated around $45 \mathrm{~F}$.

The shape of the positive EIS diagram (Fig. 5) is very different from the negative one. A classical Randles circuit or a circuit with a limited number of parameters cannot simulate its impedance properly. To estimate the double layer capacitance of the positive electrode, a diagram at colder temperature was used for which a semi-circle appears. The double layer capacitance of the positive electrode is estimated around $450 \mathrm{~F}$ in that condition, and this value is supposed to be independent of the temperature.

Evolutions of the EIS diagrams of both positive and negative electrode are shown in Figures 6 and 7. The shape of the EIS diagram of the positive electrode changes very few between $\mathrm{SoC}=30 \%$ and $90 \%$. For $\mathrm{SoC}=30 \%$, the low frequency part of the diagram becomes a vertical capacitive line. The diameter of the medium frequency semi-circle of the negative electrode EIS diagram decreases between $\mathrm{SoC}=90 \%$ and $40 \%$. A second semi-circle appears when the element is completely discharged. For both evolutions presented in Figures 6 and 7, the study of the evolution of the $R_{H F}$ is not 


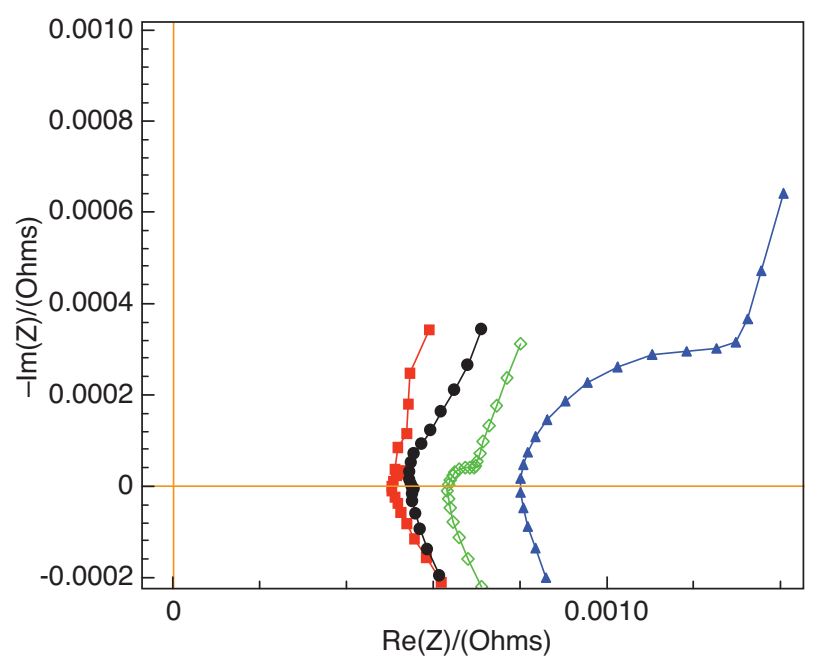

Figure 5

EIS diagram of a negative electrode at $\mathrm{SoC}=50 \%,-20^{\circ} \mathrm{C}$ (blue triangles), $0^{\circ} \mathrm{C}$ (green diamonds), $20^{\circ} \mathrm{C}$ (black circles) and $35^{\circ} \mathrm{C}$ (red squares).

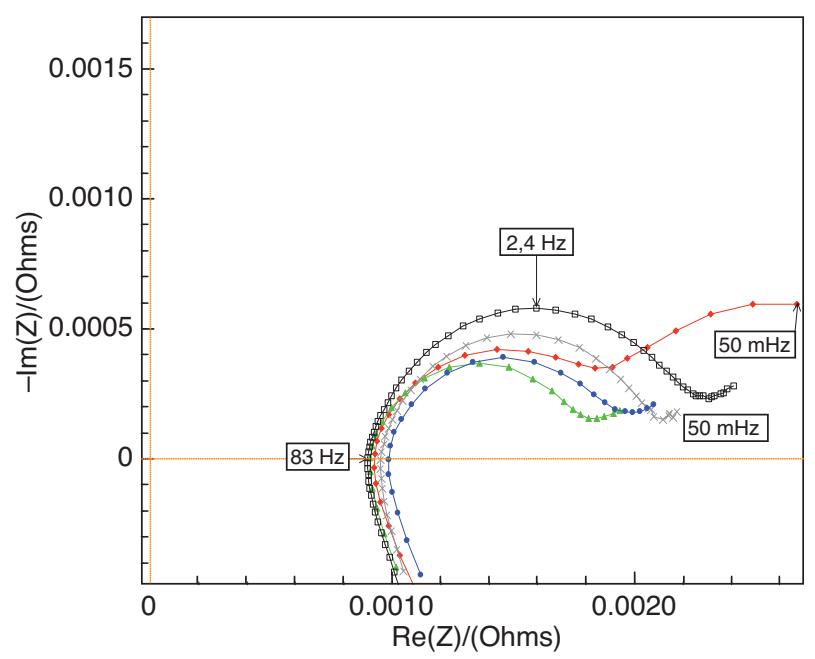

Figure 7

EIS diagram of the negative electrode of a NiMH cell at SoC $=0 \%$ (red diamonds), $20 \%$ (blue circles), $40 \%$ (green triangles), $80 \%$ (grey crosses) and $90 \%$ (black squares).

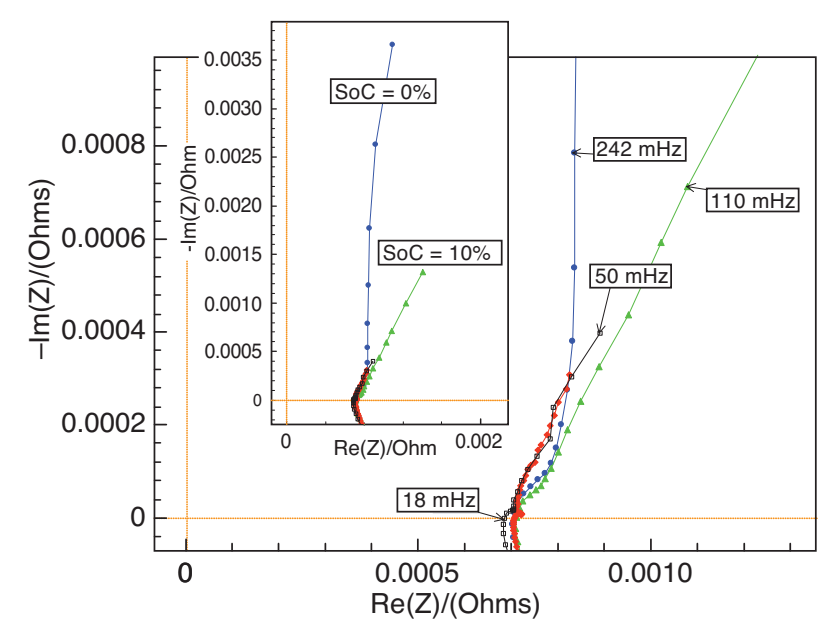

Figure 6

EIS diagram of the positive electrode of a NiMH cell at $\mathrm{SoC}=0 \%$ (blue circles), $10 \%$ (green triangles), $30 \%$ (black squares) and $90 \%$ (red diamonds).

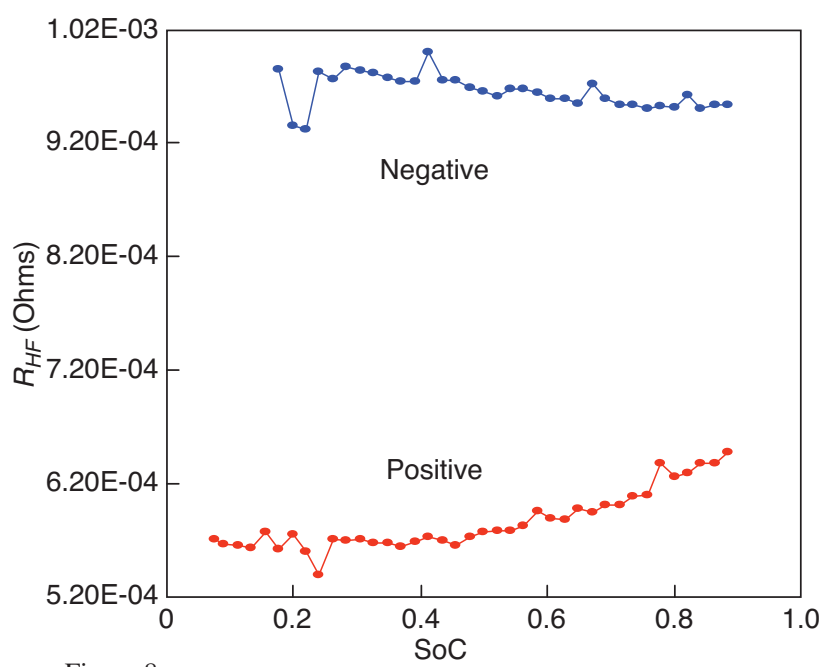

Figure 8

Evolution of the $R_{H F}$ of the positive and the negative electrode of a Ni-MH commercial cell with the SoC, $T=20^{\circ} \mathrm{C}$. possible because the connections between the potentiostat and the cell must be changed between each measurement. The lack of reproducibility induced by resistances of the cell connections disturbs the measurement of very low $R_{H F}$ resistance.

To study the contribution of each electrode on the $R_{H F}$ a measurement of the high frequency part of this diagram was done during a discharge. Figure 8 shows that the $R_{H F}$ of each electrode are not much sensitive to the SoC. The negative $R_{H F}$ higher than the positive $R_{H F}$ shows a light decrease whereas the positive $R_{H F}$ increases with increasing SoC. In a first approximation, the $R_{H F}$ of the entire cell will be considered constant in the model.

\subsection{Simulation}

On the basis of the equations described above, an advanced lumped-parameter electrochemical model has been developed in the AMESim simulation environment, using the AMESet programming tool. This battery model is aimed at being used (as a component) in the IFP simulator for hybrid electric vehicles (Fig. 9a). 


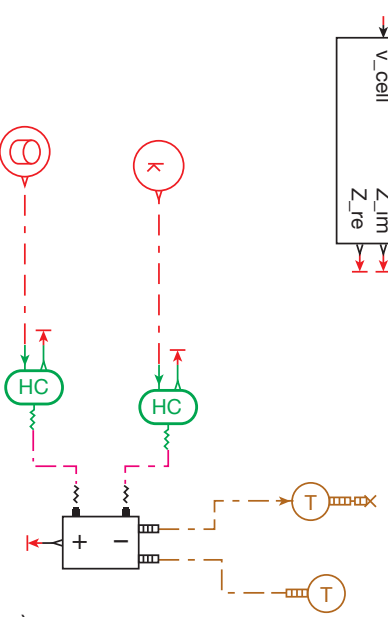

a)

Figure 9

Simulation sketches for Ni-MH cell cycling a) and for EIS b) in AMESim simulation platform.
In order to apply the EIS to the battery model executed in the AMESim platform, a special simulation technique was also developed. This technique consists of running simultaneously (co-simulation) two interexchanging models in AMESim and, respectively, Matlab, platforms. The Matlab model introduces a modulated frequency signal in the cell current during charge/discharge and it calculates the real and imaginary parts of the impedance for each frequency. In the EIS simulator the number of periods for each frequency can be set to ensure the stability of the system. The EIS simulator is able to reproduce the same test cases than the experimental EIS test bench (Fig. 9b).

\subsection{Model Parameter Adjustment}

As explained in Section 3.1, cells were characterised in terms of capacity and physical macroscopic characteristics to provide numerical parameters to the model. Electrical characteristics such as cell internal resistance and double-layer capacitance for each electrode were also measured as described in Section 2. Since the numerical values for the active material loading were not available for the Ni-MH cell under study, some physical parameters were tuned using two experimental measurements: an EIS diagram at a $60 \% \mathrm{SoC}$ and a discharge/ charge/discharge profile at $C / 2$ rate.

The EIS diagram for the cell at $60 \% \mathrm{SoC}, 20^{\circ} \mathrm{C}$ permits to adjust the parameters related to the specific surface area and the double-layer capacity per surface unit, $C_{(1)}$ and $C_{(3)}$, in such a way that the products of electrode specific surface areas and their respective $C_{(\mathrm{i})}$ 's equal the capacitance values experimentally determined. The resulting fitted EIS diagram is shown in Figure 10. A good qualitative and quantitative

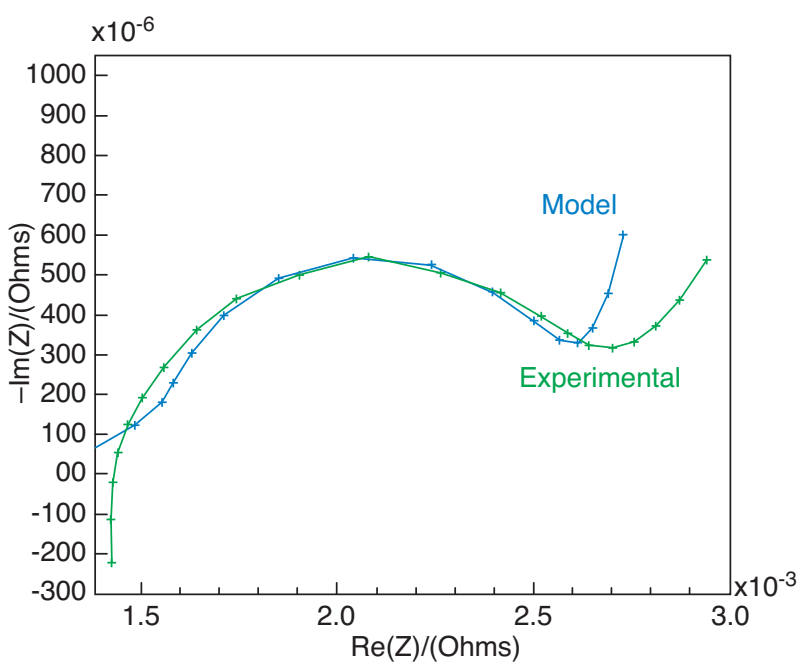

Figure 10

Comparison of the EIS diagram of a Ni-MH cell at a SoC of $60 \%$ and $20^{\circ} \mathrm{C}$ and the simulated diagram.

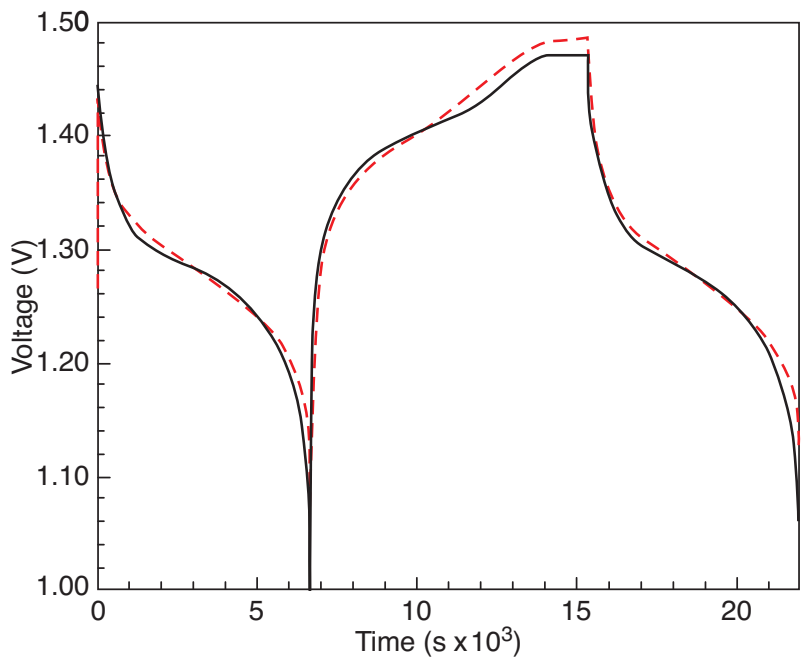

Figure 11

Comparison of the cell voltage of a Ni-MH cell during $C / 2$ rate charge/discharge cycles (black) and the fitted model (red).

agreement between model and measurements is clearly observable in the frequency range corresponding to the semi-circle.

A charge/discharge profile was used to adjust the parameters related to the electrode potentials and the hysteresis phenomenon. The resulting simulated voltage curve matches very precisely with the experimental curve, as shown in Figure 11.

\subsection{Model Evaluation}

To provide experimental validation of the model developed and tuned as described above, two different dynamic profiles were used. 


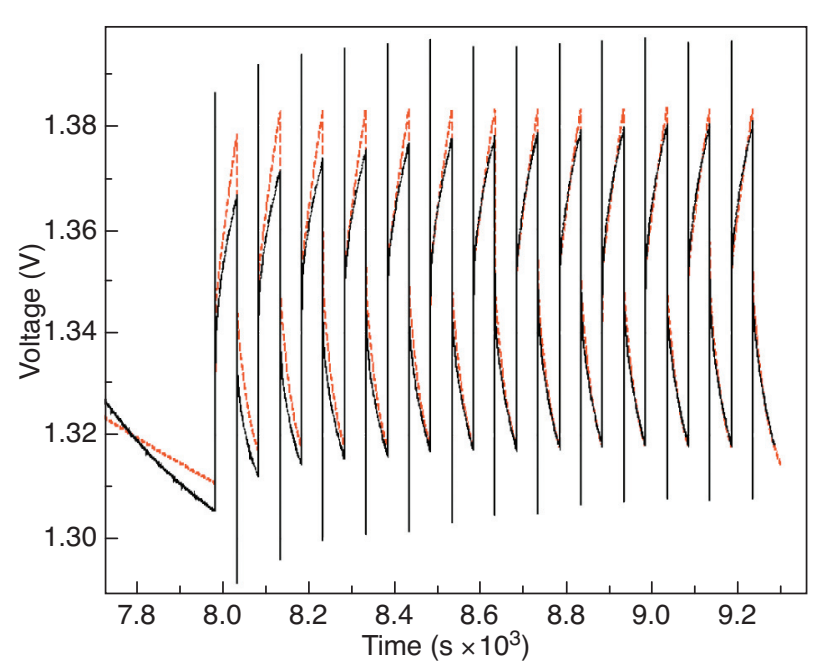

Figure 12

Comparison of the cell voltage of a Ni-MH cell during $\mathrm{C}$ rate cycling (black) with the simulation (red).

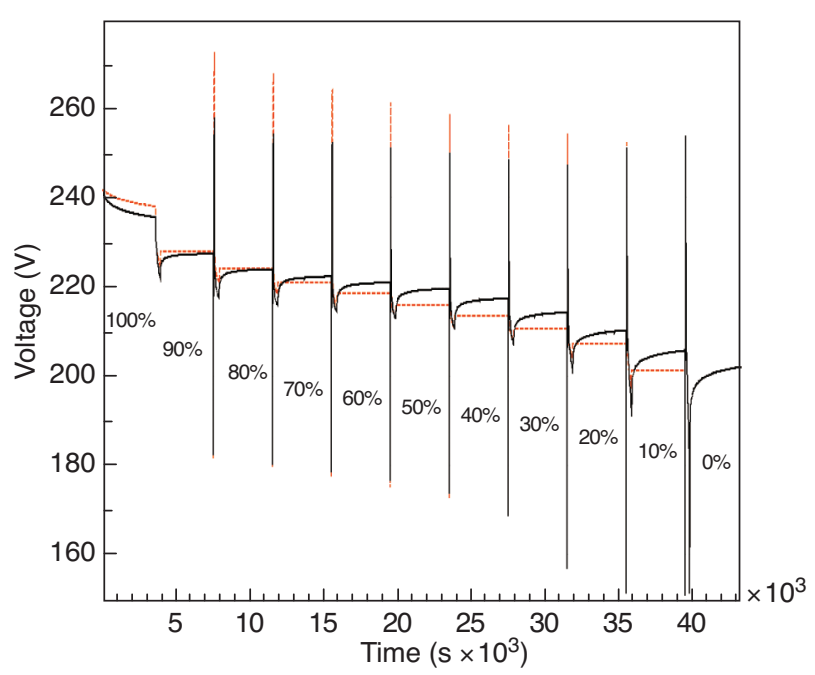

Figure 14

Comparison of measured (black line) and simulated (red dots) Ni-MH battery pack voltage during a HPPC test with theoretical SoCs.

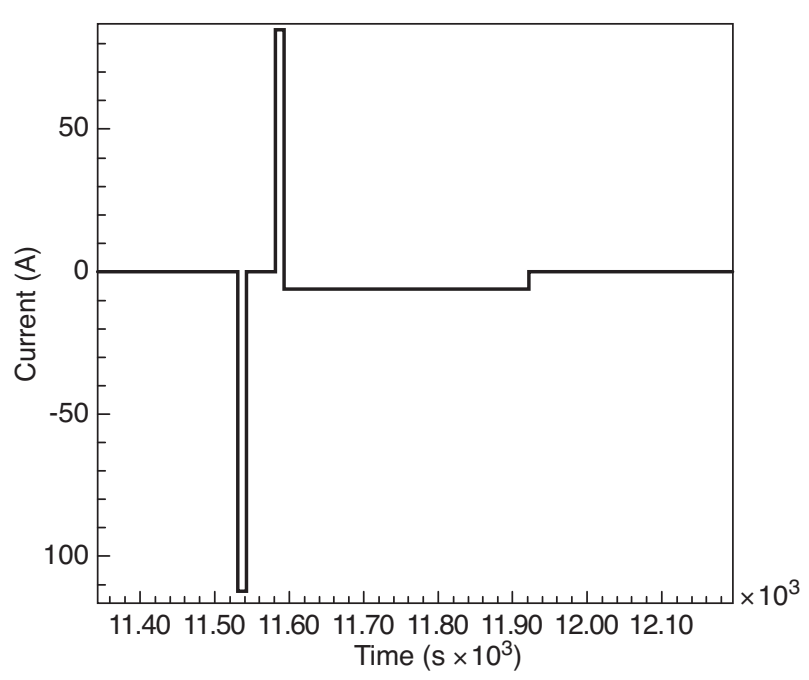

Figure 13

High Hybrid Pulse Power Characterization test profile.

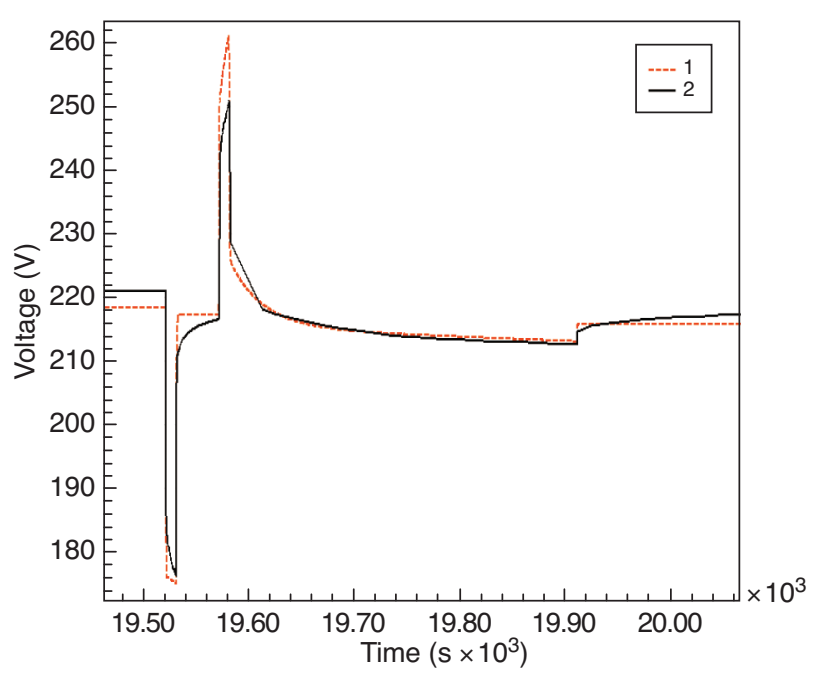

Figure 15

Comparison of measured (black line)and simulated (red dots) $\mathrm{Ni}-\mathrm{MH}$ battery pack voltage during a HPPC profile at $\mathrm{SoC}=60 \%$.
The former was applied to a single cell after discharge to a $80 \%$ SoC. It is composed of an alternation of $1 \mathrm{C}$ rate charge and discharge steps lasting $50 \mathrm{~s}$ each (Fig. 12). It should be noted that the "infinite" voltage values (vertical lines) visible on the experimental curve are due to the potentiostat commuting between two current ranges. The figure clearly shows a qualitative correspondence of the simulated and experimental curves. A quantitative analysis of differences reveals that the discrepancy does not exceed $\pm 1.8 \%$ of the operating range of the NiMH cell under these cycling conditions.
The second validation test presented is a HPPC test performed at $20^{\circ} \mathrm{C}$ for the entire battery pack. The pack consists of 168 serially connected cells. However, it is assumed that cell construction, SoC, and temperature are uniform throughout the pack. Thus no attempt was made to account for cell-to-cell differences arising from manufacturing variability or temperature distribution within the pack. The complete HPPC test consists with a succession of sequences as shown in Figure 13. Figure 14 gives the results for the complete HPPC test and Figure 15 displays the result for a single profile. 
The model predictions in these highly dynamic conditions reflect the experimental measurements quite correctly over the nine periods of the HPPC test. However, there is an increasing difference in the voltage at the end of the rest period with increasing discharge/charge pulses, suggesting that the relaxation phenomena could be better taken into account in the model. Nevertheless model prediction can be still considered as fairly good in the relaxation periods, since the relative error between measurements and simulation remains below $\pm 3.8 \%$ of the operating range of the $\mathrm{NiMH}$ battery pack. Notice however that discharge pulses are better reproduced than charge pulses, for which the difference reaches $9 \%$ after the fourth pulse.

\section{DISCUSSION}

Generally speaking, the improved OD model of a sealed $\mathrm{Ni}-\mathrm{MH}$ cell and battery pack is validated at $20^{\circ} \mathrm{C}$ as far as voltage prediction is concerned. Additional work is in progress, focusing on the thermal aspects of the modelling in conditions representative of actual operation.

It is now interesting to discuss the model predictions in terms of SoC with regards to the conventional Coulomb counting method during the HPPC test. The initial state of the battery was set to $100 \%$ SoC. After the eight pulse where the SoC theoretically equals $10 \%$, it is observed in the experimental curve (Fig. 16) that the discharge pulse was limited by the low-voltage limit of the battery, suggesting that less than $10 \%$ of charge was remaining in the battery. Figure 16 compares the SoC simulated with the 0D electro-

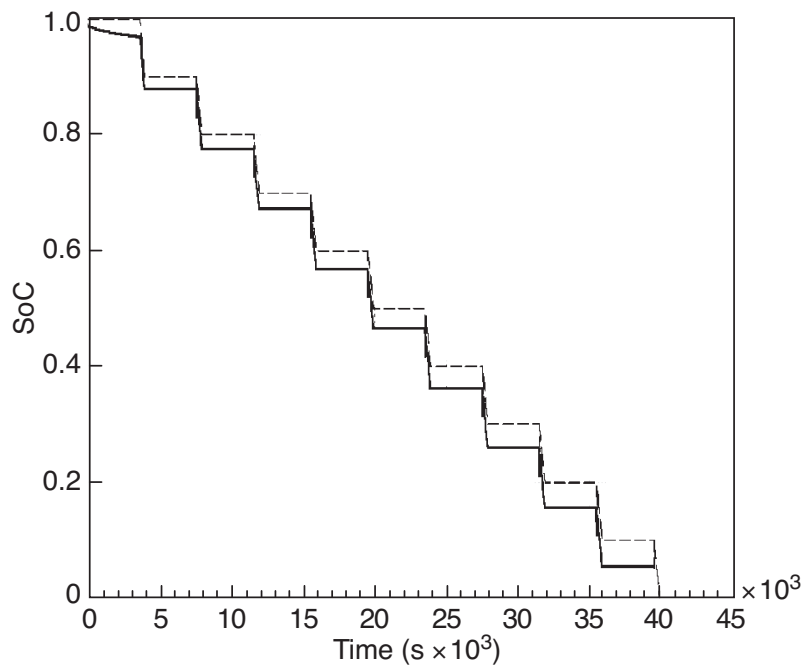

Figure 16

Comparison of simulated SoC evolution (line) and $\mathrm{SoC}$ evolution obtained with coulomb counting method (dot line) during a complete HPPC test. chemical model with SoC calculated using the Coulomb counting method. Focusing on the model prediction after the eight pulse, for example, the estimated $\mathrm{SoC}$ value using Equation (25) is around 5\%, which is in agreement with the aforementioned observation. This is indeed a very good result in our scope of work where this model would be used with confidence as a SoC observer within a BMS system.

\section{CONCLUSION}

Electrochemical lumped-parameter models based on the cell electrochemistry appear as very promising mathematical models of the battery systems to provide reliable monitoring within the vehicle management system. Indeed the SoC is calculated from the concentration of the reactants, which are dynamic states updated with mass and current balance equations. The direct benefit of this approach, using a model that includes the SoC as a state, is that a Kalman filter or an equivalent mathematical tool can automatically give a dynamic estimate of the $\mathrm{SoC}$ and its uncertainty. Moreover, the computational time needed to solve such simplified physics-based battery models is well adapted for online applications.

The improved OD model of a sealed Ni-MH cell/battery presented in this paper shows a good agreement with measurements performed for low rate solicitations on a single cell but also for high rate solicitations like HPPC test on a entire battery pack. Work is in progress to adjust the thermal parameters of the model and provide a complete voltage/thermalbehaviours battery model.

Future development of the model will take into account the ageing phenomena occurring in the battery during service operation.

\section{REFERENCES}

1 Guzzella L., Sciarretta A. (2005) Introduction to Modeling and Optimization in Vehicle Propulsion Systems, Springer, Berlin, Heidelberg.

2 Plett G.L. (2004) Extended Kalman filtering for battery management systems of LiPB-based HEV battery packs: Part 1 Background, J. Power Sources 134, 252-261.

3 Piller S., Perrin M., Jossen A. (2001) Methods for state-ofcharge determination and their applications, J. Power Sources 96, 113-120.

4 Pop V., Bergveld H.J., Notten P.H.L., Regtien P.P.L. (2005) State-of-the-art of battery state-of-charge determination, Meas. Sci. Technol. 16, R93-R110.

5 Pop V., Danilov D., Bergveld H.J., Notten P.H.L., Regtien P.P.L. (2006) Adaptative state-of-charge indication system for Li-ion battery powered vehicle, The 22nd International Battery, Hybrid and Fuel Cell Electric Vehicle Symposium \& Exposition, Yokohama, Japan, 23-10-2006. 
6 Pop V., Bergveld H.J., Op het Veld J.H.G., Regtien P.P.L., Danilov D., Notten P.H.L. (2006) Modeling battery behavior for accurate state-of-charge indication, J. Electrochem. Soc. 153, A2013-A2022.

7 Thele M., Buller S., Sauer D.U., De Doncker R.W., Karden E. (2005) Hybrid modeling of lead-acid batteries in frequency and time domain, J. Power Sources 144, 461-466.

8 Kuhn E, Forgez C., Friedrich G. (2004) Modeling diffusive phenomena using non integer derivatives Application $\mathrm{NiMH}$ batteries, Eur. Phys. J.-Appl. Phys. 25, 183-190.

9 Kuhn E., Forgez C., Lagonotte P., Friedrich G. (2006) Modelling Ni-mH battery using Cauer and Foster structures, J. Power Sources 158, 1490-1497.

10 Takano K., Nozaki K., Saito Y., Negishi A., Kato K., Yamaguchi Y. (2000) Simulation study of electrical dynamic characteristics of lithium-ion battery, J. Power Sources 90, 214-223.

11 Thele M., Bohlen O., Sauer D.U., Karden E. (2008) Development of a voltage-behavior model for NiMH batteries using an impedance-based modeling concept, J. Power Sources 175, 635-643.

12 Paxton B., Newman J. (1997) Modeling of nickel/metal hydride batteries, J. Electrochem. Soc. 144, 3818-3831.

13 Gu W.B., Wang C.Y., Li S.M., Geng M.M., Liaw B.Y. (1999) Modeling discharge and charge characteristics of nickel-metal hydride batteries, Electrochim. Acta 44, 4525-4541.

14 Wu B., Mohammed M., Brigham D., Elder R., White R.E. (2001) A non-isothermal model of a nickel-metal hydride cell, $J$. Power Sources 101, 149-157.

15 Wu B., Dougal R., White R.E. (2001) Resistive companion battery modeling for electric circuit simulations, J. Power Sources 93, 186-200.

16 De Vidts P., Delgado J., White R.E. (1995) Mathematical Modeling for the Discharge of a Metal Hydride Electrode, $J$. Electrochem. Soc. 142, 4006-4013.
17 Botte G.G., Subramanian V.R., White R.E. (2000) Mathematical modeling of secondary lithium batteries, Electrochim. Acta 45, 2595-2609.

18 Ning G., White R.E., Popov B.N. (2006) A generalized cycle life model of rechargeable Li-ion batteries, Electrochim. Acta $\mathbf{5 1}$, 2012-2022.

19 Santhanagopalan S., White R.E. (2006) Online estimation of the state of charge of a lithium ion cell, J. Power Sources 161, 1346-1355.

20 Zhang Q., White R.E. (2007) Comparison of approximate solution methods for the solid phase diffusion equation in a porous electrode model, J. Power Sources 165, 880-886.

21 Sciarretta A., Sauvant-Moynot V., Faille I. (2008) Advances in model-based SoC determination for HEV traction batteries, $A E A$ 2008, 4th European Conference on Alternative Energies for the Automotive Industries, paper 13.

$22 \mathrm{Gu}$ W.B., Wang C.-Y. (2000) Thermal-electrochemical Modeling of Battery systems, J. Electrochem. Soc. 147, 2910-2922.

23 Huet F. (1998) A review of impedance measurements for determination of the state-of-charge or state-of-health of secondary batteries, J. Power Sources 70, 59-69.

24 FreedomCar (2003) FreedomCar Battery Test Manual For Power-assist hybrid Electric vehicle, INEEL/DOE.

25 Gu W.B., Wang C.Y., Liaw B.Y. (1998) Micro-macroscopic coupled modeling of batteries and fuel cells, J. Electrochem. Soc. 145, 3418-3427.

26 Newmann J., Thomas-Alyea K.E. (2004) Electrochemical systems, 3rd ed., John Wiley \& Sons, New York.

Final manuscript received in August 2009 Published online in November 2009 